\title{
Fragonard, Marie-Madeleine.
}

\section{Variations sur la grâce et l'impuissance de la parole.}

Paris: Presses Sorbonne Nouvelle, 2011. 2 tomes, 313 et 316 p. ISBN 978-287854 et 978-2-87854-509-8 (broché), $25 €$

Les quarante articles de M.-M. Fragonard qui forment les deux volumes de cet ouvrage sont des textes qui ont déjà paru, mais qui ont été regroupés dans l'intérêt du thème abordé, et élucidé dans le beau titre qui les englobe. Ils montrent, en effet, à la fois la fragilité et la nécessité de la parole en temps de trouble, la parole telle qu'elle a fonctionné dans le cadre d'une rhétorique religieuse dominante à l'époque étudiée, celle d'Henri IV et notamment dans les années 1590-1620. Comme l'explique Fragonard, à l'exception de quelques auteurs bien connus (Rapin, Pasquier, Aubigné), la littérature de cette époque est fort peu étudiée et éditée alors que son rôle est fondamental du fait même qu'elle permet de dater l'avènement des nouvelles formes marquant les domaines esthétique, historique ou politique.

Les deux tomes sont organisés en trois volets. Le premier volet intitulé "La mouvance des formes littéraires ", rassemble des articles qui montrent que le mot baroque ne traduit pas de manière adéquate tout ce qui constitue la représentation littéraire de cette époque. Malgré ses connotations d'instabilité et de mobilité, ce terme ne permet ni de situer chronologiquement le virage qui annonce la fin du XVI ${ }^{e}$ siècle, ni de tenir compte de la naissance d'une multitude de formes littéraires. Or ces formes brouillonnes, sans noms spécifiques, dégradent les formes préexistantes et en fondent de nouvelles qui, comme c'est le cas de la pastorale ou du roman réaliste, seront élaborées et régularisées plus tard. Ainsi, le récit romanesque qui s'épanouit au XVII siècle apparait à l'état embryonnaire dans le poème héroïque La Néréide de Pierre de Deimier qui, lui, suit le modèle canonique créé par l'Arioste, mais en le subvertissant par l'introduction d'une histoire sentimentale.

C'est à travers l'analyse d'œuvres aujourd'hui oubliées que Fragonard parvient à montrer comment, en temps de guerre, la littérature devient un refuge qui, à long terme, aboutit à une littérature autonome, indépendante des pouvoirs politique et religieux, même si, à court terme, cette littérature relève plus de l'ordre du repli ou de la polémique, comme l'indique le titre du second volet. La polémique est ce qui révèle la puissance de la parole et le règne d'Henri IV est précisément une période où les gens de lettres utilisent les circonstances 
historiques pour mettre en scène la haine ou la peur de l'autre, et cela d'une manière d'autant plus efficace et troublante qu'ils agissent sous le couvert de la science (dans un dictionnaire d'hérésies), du ridicule (dans des pamphlets et des mazarinades) ou de l'imagination (par le biais de métaphores). Inquiétants, car, pour prendre l'exemple du dictionnaire d'hérésies, figer un état linguistique implique une certaine représentation du monde et surtout une définition univoque d'un certain groupe : ainsi des Luthériens qui, tout en traitant les Calvinistes de Calvinistes, les traitaient aussi de Turcs, de Musulmans, de poisons ; en retour, les Calvinistes traitaient les Luthériens d'Ubiquistes, de Semi-Papistes et de buveurs de sang. Alarmants aussi, ces écrits de combats, car, comme le souligne la métaphore de l'hérétique en singe élaborée par Saconay, même si cette métaphore est une fiction et ressort de la fable, elle agit toujours sur le lecteur, d'autant plus que, suivant l'analyse attentive de Fragonard, il n'y a rien à décrypter et donc qu'elle dit ce qu'elle dit, à savoir " tuez-les tous » (p. 165), soit tuez tous les hérétiques que ce soit d'un côté ou de l'autre. Comme l'explique Fragonard dans le troisième volet, les auteurs de ce temps vivent une histoire qui les contraint à prendre parti et, pour cette raison, l'éthique a un rôle à jouer non seulement au présent, mais aussi dans la reconstruction du passé. Ils gèrent des faits obscurcis par une parole destinée à agir sur le public, mais leur démarche de retrouver la vérité historique laisse à désirer, car elle paraît souvent subordonnée à l'intention, non pas d'informer mais celle de bouleverser le lecteur contemporain. Que l'on songe au projet critique des érudits, tels que Gallonius ou Baronius, qui revisitent l'Antiquité pour tenter de dégager la signification de mots qui ont perdu leurs référents, entre autres celle des instruments qui servaient dans l'Antiquité au supplice des martyrs.

Le second volume regroupe des textes qui, plus visiblement inspirés de discours théologiques et de situations religieuses et philosophiques, de même que guidés par un désir d'unité, exposent le maniement de la parole pour négocier le réel, la matière ou le concret, mais qui transmettent néanmoins l'idée du spirituel, du surnaturel. D'où la mise en relief par Fragonard de l'utilisation importante par les auteurs de tropes et de figures, telles que l'allégorie, la métaphore ou l'analogie. Ces procédés font coexister deux pans de réalités : le surnaturel ou le sacré. C'est ce que montre l'article sur l'œuvre de d'Aubigné, laquelle présente la ville de Paris en véritable Babel ensanglantée. Dans le volet intitulé " Matière et esprit », les analyses de Fragonard révèlent combien l'opposition entre imagination et réalité, visible et invisible, parole 
humaine et parole divine est une opposition fragile et précaire. Ainsi, l'article portant sur les Essais - où Montaigne affirme que la maladie et la douleur sont le prix qu'il faut payer pour avoir une garantie de l'existence des choses - fait voir que cette attitude du philosophe amène en fait à douter de la réalité des choses, puisque celles-ci n'ont plus de valeur en soi mais seulement par rapport à la valeur qu'on y met. La difficulté de différencier entre illusion et réel est reprise dans l'article "Corps simulés, corps simulateurs » mettant en relief le découragement d'Amboise Paré face à une science et à la nature qui jouent sur l'équivoque et les ressemblances. L'article examine l'aveu du médecin, selon qui les maladies supposées venir du diable ne peuvent pas être guéries par les médecins, mais en retour les diables ou les sorciers ne peuvent pas guérir les maladies naturelles. Le dernier volet de l'ouvrage l'explique bien ; c'est précisément parce que la rhétorique n'est pas seulement affaire de technique et d'esthétique, mais comporte un contenu, qu'il est important de négocier, et surtout de négocier avec ce qui semble le moins négociable, à savoir l'emprise du religieux sur les âmes. À cette époque, cette emprise est de moins en moins influencée par les textes religieux puisque, comme le suggère Fragonard, c'est surtout à travers la littérature que s'accomplit cette négociation. La fiction est plus envoûtante et séductrice qu'un sermon, même si les modèles de pitié ou de sainteté qui y sont mis en scène semblent moins écrits pour encourager les lecteurs - surtout les lectrices - à les imiter qu'à les repousser, ou du moins à s'en distancier. Qu'on lise La Madeleine au désert de Pierre de Saint-Louis, qui se livre à défarder de manière brutale les coquettes mondaines, ou encore la nouvelle Floriane de Jean-Pierre Camus, qui s'indigne face au désordre que représente le mélange entre vie de tous les jours et dévotion. Ceci est encore plus évident dans la nouvelle tragique de François de Rosset sur le curé Gaufridy. Ce récit met en scène un diable qui prêche les vertus de la foi chrétienne, effectuant ainsi un renversement total de la compréhension du diabolique et par-là du sacré, surtout que cela semble se faire avec la permission de Dieu. Mais comment savoir si c'est Dieu lui-même qui parle à travers le diable ou le prédicateur? Dans son dernier article, Fragonard aborde le problème de la rhétorique sacrée en montrant que l'efficacité de celle-ci réside dans sa sincérité qui est à la fois appel à l'action de l'esprit Saint et reconnaissance d'une parole humaine impuissante ou inadéquate face à la situation de la croyance. Le second volume se ferme sur une bibliographie des articles publiés de Fragonard et un index de noms, fort utile, puisqu'il s'agit d'auteurs peu connus. Tous les articles 
réunis dans les deux volumes font apparaître le travail érudit, minutieux, patient et passionné d'une universitaire, chercheure et enseignante, dont la lecture enrichit le lecteur. Fragonard parvient à l'enthousiasmer, car au savoir du spécialiste, elle mêle un ton varié qui passe du sérieux au léger et vice versa, et qui souvent laisse transparaître le lien intime et affectif qu'elle entretient avec ses auteurs. Ces deux volumes qui réunissent des articles auparavant dispersés sont une contribution importante aux études de la période d'Henri IV et sont destinés aux spécialistes des XVI ${ }^{\mathrm{e}}$ et XVII ${ }^{\mathrm{e}}$ siècles, mais aussi à tous ceux qui veulent mieux comprendre le lien entre littérature, vie et parole.

AGNES CONACHER, Queen's University

\section{Hiscock, Andrew.}

\section{Reading Memory in Early Modern Literature.}

Cambridge, UK: Cambridge University Press, 2011. Pp. xi, 320. ISBN 978-0521-76121-5 (hardcover) \$87.

What is admirable about this study is its comprehensive coverage of the traditions and operations of memory in Tudor and early Stuart English literature. Its ambitious breadth can be discerned not only in the wonderfully eclectic passages on remembering the past that Hiscock has gathered from unexpectedly germane corners of early modern textuality, but more significantly in its coverage of a wide selection of notable English authors whose writings revolve closely around questions of all things memorial. Rather than concentrate on a few playwrights or poets within a narrow generic band, Hiscock's study investigates a range of genres and literary discourses, all eight chapters but one focusing on a single seminal author with a distinctive generic or multi-generic outlook.

The first chapter deals with the ways in which Surrey's courtly and erotic poetry deploys acts of memory for critiquing the culture of the Tudor court. Chapter 2 examines how Katherine Parr's prayers and meditations constitute memory as a political act during Henry's later years when reformed attitudes coexisted uneasily, even dangerously, with established Catholic doctrines. The third chapter explores the multiple roles that commemoration play in 